\title{
Band structures of plasmonic polarons
}

\author{
Fabio Caruso, Henry Lambert, and Feliciano Giustino \\ Department of Materials, University of Oxford, Parks Road, Oxford OX1 3PH, United Kingdom
}

(Dated: October 15, 2018)

\begin{abstract}
Using state-of-the-art many-body calculations based on the ' $G W$ plus cumulant' approach, we show that electron-plasmon interactions lead to the emergence of plasmonic polaron bands in the band structures of common semiconductors. Using silicon and group IV transition metal dichalcogenide monolayers $\left(\mathrm{AX}_{2}\right.$ with $\mathrm{A}=\mathrm{Mo}, \mathrm{W}$ and $\left.\mathrm{X}=\mathrm{S}, \mathrm{Se}\right)$ as prototypical examples, we demonstrate that these new bands are a general feature of systems characterized by well defined plasmon resonances. We find that the energy vs. momentum dispersion relations of these plasmonic structures closely follow the standard valence bands, although they appear broadened and blueshifted by the plasmon energy. Based on our results we identify general criteria for observing plasmonic polaron bands in the angle-resolved photoelectron spectra of solids.
\end{abstract}

Electron-boson interactions are pervasive in manybody physics and the resulting quasiparticles are clear examples of emergent behavior in quantum matter. While in the case of phonons and magnons the fingerprints of their interactions with electrons in angle-resolved photoemission spectra (ARPES) are largely understood [1, much less is known about electron-plasmon interactions. A detailed and quantitative description of these interactions is key to refine our understanding of electronic excitations in condensed matter, and could provide new pathways towards plasmon-assisted band-gap tuning [2, or the manipulation of plasmon polaritons, with potential implications for photonics and plasmonics [3].

In ARPES, the acceleration of a photo-electron upon photon absorption may trigger shake-up excitations in the sample, leading to the emission of phonons, electronhole pairs, and plasmons, the latter being collective charge-density fluctuations 4. Intuitively, if a photon excites both a hole and a plasmon, the ARPES signal should exhibit spectral weight at energies corresponding to the sum of the binding energy of the electron and the excitation energy of the plasmon, as obtained for example from electron energy loss spectroscopy (EELS) [5]. This phenomenology is analogous to the emergence of 'peakdip-hump' structures in ARPES as a result of electronphonon interactions 1, 6-11; the difference between the resulting spectral features arises from the characteristic energy of the boson $(\sim 10 \mathrm{meV}$ for phonons, $\sim 10 \mathrm{eV}$ for plasmons). In fact the very first model of electronplasmon interactions [4, 12, 13] is formally equivalent to the electron-phonon Hamiltonian developed for the polaron problem [14. In this model, the electron-plasmon interaction results in 'plasmonic polarons' [15, in complete analogy with the polarons of the ordinary theory of electron-phonon interactions [16].

Identifying plasmonic polarons in ARPES spectra is notoriously difficult. While plasmonic satellites have been successfully identified in the integrated photoemission spectra of $\mathrm{Na}$ [17] and $\mathrm{Si}[18$, the identification of energy vs. momentum dispersions of plasmonic polarons in angle-resolved spectra has proven considerably more chal- lenging [19]. So far such dispersions have been observed only in the case of graphene, and only in a narrow region of the Brillouin zone around the Dirac point [20, 21]. Key factors hindering the observation of the dispersion relations of plasmonic polarons are (i) the energy scale of the plasmon energy, which requires using energetic photons at the expense of momentum resolution; (ii) the increased phase-space for electron-phonon scattering and electronhole pair generation, which adds to the spectral broadening; and (iii) the possible mix up of weak plasmonic satellites and strong quasi-particle peaks.

In this work we perform state-of-the-art first-principles calculations to show that electron-plasmon interactions lead to the formation of plasmonic-polaron bandstructure replica. These new structures appear as broadened copies of the valence bands shifted by the plasmon energy. Using a combination of many-body perturbation theory in the $G W$ approximation 2224 and the cumulant expansion approach [4, 13, 17, 18, 25) 29. we demonstrate the presence of plasmonic-polaron bands in silicon. We further show that two-dimensional group IV transition-metal dichalcogenides (TMDs) $\mathrm{AX}_{2}$ with $\mathrm{A}=\mathrm{Mo}, \mathrm{W}$ and $\mathrm{X}=\mathrm{S}, \mathrm{Se}$ [30, 31] provide an ideal playground for the experimental observation of these novel spectroscopic signatures of the electron-plasmon coupling.

Within the sudden approximation, the photocurrent measured in ARPES experiments is proportional to the electron spectral function $A(\mathbf{k}, \omega)[1,32$, where $\mathbf{k}$ is the crystal momentum of the electron and $\omega$ its binding energy (here and in the following atomic units are understood). The spectral function can be calculated by using the cumulant expansion [4, 13, 17, 18, 26]: the electron Green's function is expanded in terms of the screened Coulomb interaction $W$, and a subset of diagrams is evaluated to all orders of perturbation [4]. This strategy leads to a more accurate treatment of dynamical correlation as compared to the standard $G W$ approximation 22 . The cumulant expansion draws from the exact solution of the polaron problem [16, and was originally applied to study plasmon satellites in core-level spectra [13]. Importantly, 
it is also valid in the case of valence electrons, as the effects of electron recoil (change of electron momentum) upon plasmon emission tend to cancel out 4.

In this work we use the formulation of the cumulant expansion given by 4 and [17, which we will refer to as the $G W+\mathrm{C}_{\mathrm{AHK}}$ approach. In this formulation only the first cumulant is retained in order to describe the lineshape of quasi-particles and one-plasmon excitations. The case of $n$-plasmon excitations $(n \geq 2)$ is of little interest here since the corresponding spectral signatures are damped by the Lang-Firsov factor $a^{n} / n$ !, with $a$ the average number of plasmons around the hole [13]. The $G W+\mathrm{C}_{\mathrm{AHK}}$ spectral function can be expressed as [17]:

$$
A(\mathbf{k}, \omega)=\sum_{n}\left[A_{n}^{\mathrm{QP}}(\mathbf{k}, \omega)+A_{n}^{\mathrm{QP}}(\mathbf{k}, \omega) A_{n}^{\mathrm{C}}(\mathbf{k}, \omega)\right] .
$$

Here $A_{n}^{\mathrm{QP}}$ denotes the quasi-particle contribution to the $G_{0} W_{0}$ spectral function:

$$
A_{n}^{\mathrm{QP}}(\mathbf{k}, \omega)=\frac{1}{\pi} \frac{\left|\Sigma_{n \mathbf{k}}^{\prime \prime}\left(\varepsilon_{n \mathbf{k}}\right)\right|}{\left[\omega-\varepsilon_{n \mathbf{k}}-\Sigma_{n \mathbf{k}}^{\prime}\left(\varepsilon_{n \mathbf{k}}\right)\right]^{2}+\left[\Sigma_{n \mathbf{k}}^{\prime \prime}\left(\varepsilon_{n \mathbf{k}}\right)\right]^{2}},
$$

where $\Sigma^{\prime}\left(\Sigma^{\prime \prime}\right)$ indicates the real (imaginary) part of the $G_{0} W_{0}$ self-energy [22, 23], and $\varepsilon_{n \mathbf{k}}$ the Kohn-Sham eigenvalue. In Eq. (2) it is assumed that the off-diagonal elements of the self-energy are small and can be neglected, as is typically the case [23, 33. The term $A_{n}^{\mathrm{C}}$ in Eq. (1) is defined as [17]:

$$
A_{n}^{\mathrm{C}}(\mathbf{k}, \omega)=\frac{\beta_{n \mathbf{k}}(\omega)-\beta_{n \mathbf{k}}\left(\varepsilon_{n \mathbf{k}}\right)-\left.\left(\omega-\varepsilon_{n \mathbf{k}}\right) \frac{\partial \beta_{n \mathbf{k}}}{\partial \omega}\right|_{\varepsilon_{n \mathbf{k}}}}{\left(\omega-\varepsilon_{n \mathbf{k}}\right)^{2}},
$$

where $\beta_{n \mathbf{k}}(\omega)=\pi^{-1} \Sigma_{n \mathbf{k}}^{\prime \prime}(\omega) \theta(\mu-\omega), \mu$ being the chemical potential. This term accounts for interactions between the photo-hole and one-plasmon excitations [32], and its contribution to the spectral function in Eq. (2) is to be identified with plasmonic polarons.

Using Eqs. (1)-(3), we now investigate the signatures of plasmonic polarons in silicon. In Fig. 1 (a) and (b) we report the angle-resolved spectral function of silicon obtained from the Sternheimer- $G_{0} W_{0}$ approach (SGW) and $\mathrm{S} G W$ plus cumulant $\left(\mathrm{S} G W+\mathrm{C}_{\mathrm{AHK}}\right)$, respectively. Details on $\mathrm{S} G W$ are provided in the Supplemental Material [34] and Refs. 35, 36]. For silicon, the experimental (integrated) photoemission spectrum shown in Fig. 1 (c) is characterized by a broadened plasmonic resonance covering approximately the energy range from $16 \mathrm{eV}$ to $30 \mathrm{eV}$ below the Fermi energy. In this resonance we can clearly identify three distinct structures (dashed blue lines). The bright energy bands visible in Fig. 11(a) for binding energies between 0-12 eV correspond to the standard quasiparticle peaks. These peaks result from photoionization processes occurring in absence of plasmon excitations, and define the ordinary valence band structure of silicon. In addition to the quasi-particle features, the spectral

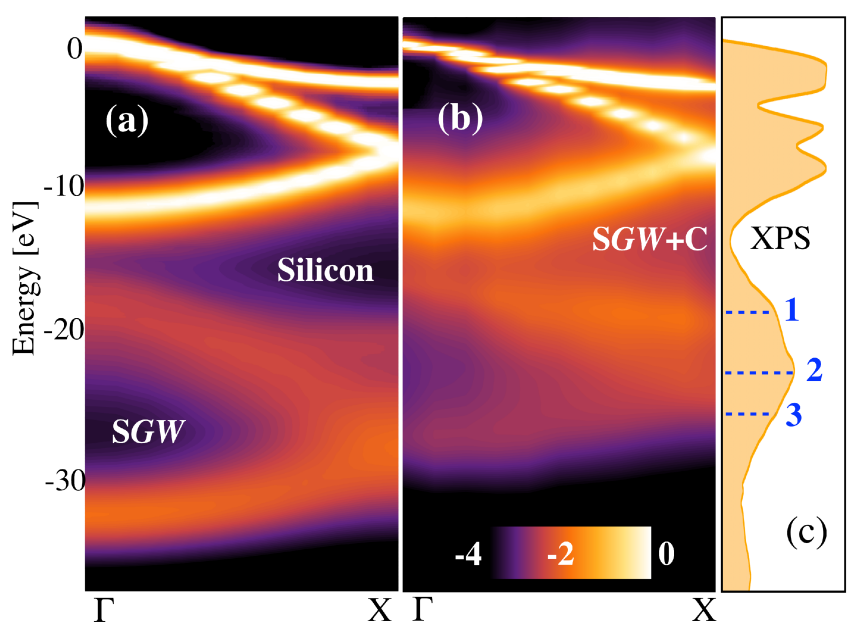

Figure 1. Angle-resolved spectral function of silicon on a logarithmic scale for wavevectors along the $\Gamma-X$ high-symmetry line, evaluated using (a) the Sternheimer- $G W$ method (SGW) and (b) the $\mathrm{S} G W$ plus cumulant $(\mathrm{S} G W+\mathrm{C})$ approach. (c) Measured X-ray photoemission spectrum of silicon (XPS) from Ref. [18. The blue dashed lines indicate the three features discussed in the main text.

function exhibits a rich structure at binding energies between 15-30 eV. These structures can be identified with plasmonic polarons. These features are present already at the $G_{0} W_{0}$ level, however, their energy range is largely overestimated and, thus, not compatible with the plasmonic features observed in XPS. The inclusion of the cumulant correction in $\mathrm{S} G W+\mathrm{C}_{\mathrm{AHK}}$ moves the plasmonic polaron resonances to lower binding energy, improving the agreement with the experimental spectrum significantly. This improvement reflects the inclusion of higherorder exchange-correlation diagrams in the $G W+\mathrm{C}_{\mathrm{AHK}}$ Green function, as discussed in more detail in the Supplemental Material 34.

Unexpectedly, plasmonic polarons exhibit dispersion relations which follow closely the ordinary band structure resulting from the quasi-particle peaks. The striking similarity between the dispersion of the valence bands and the plasmonic structures suggests that we are looking at band structures of plasmonic polarons. Plasmonic polaron bands appear as blue-shifted replicas of the ordinary valence bands, but they are considerably broader and less intense. The comparison of Fig. 1(b) and (c) suggests that the plasmon satellite of silicon [18] results from the momentum-average of plasmonic polaron bands over the first Brillouin zone. For quasiparticles, it is well known that the density of states is characterized by singularities (known as Van Hove singularities) at the energies for which the first momentum derivative of the quasiparticle bands vanishes $\left(\nabla_{\mathbf{k}} \varepsilon_{\mathbf{k}}=0\right)$. Correspondingly, peaks in the density of states can be associated with extremal points of quasiparticle bands. Figures 1(b) and 

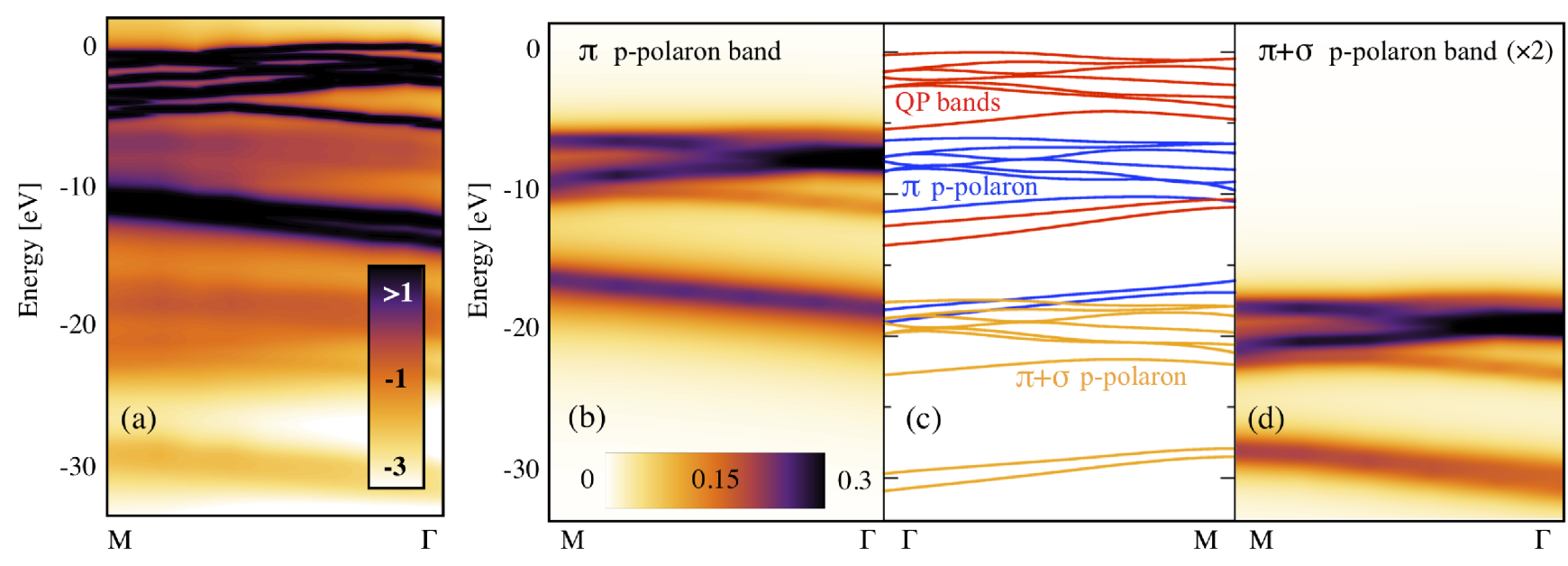

Figure 2. (a) Complete angle-resolved spectral function of monolayer $\mathrm{MoS}_{2}$ on a logarithmic scale for wavevectors along the $\Gamma-M$ high-symmetry line, evaluated using the $G W+\mathrm{C}_{\mathrm{AHK}}$ approach. (b), (d) Contributions of plasmonic polarons to the spectral function in (a): $\pi$-plasmonic polarons (b) and $(\pi+\sigma)$-plasmonic polarons (d). (c) Quasi-particle band structure of monolayer $\mathrm{MoS}_{2}$ extracted from (a) (red solid lines), band structure of $\pi$-plasmonic polarons extracted from (b) (blue solid line), and band structure of $(\pi+\sigma)$-plasmonic polarons from $(\mathrm{d})$ (yellow solid line). Energies are referenced to the valence-band top.

(c) indicate that Van Hove singularities may also have a plasmonic origin. In particular, the two experimental peaks at $20.4 \mathrm{eV}$ and $24.5 \mathrm{eV}$ and the shoulder at $27.3 \mathrm{eV}$ [labelled as 1-3 in Fig. 1(c)] can be attributed to the vanishing of the first momentum derivative of the plasmonic polaron bands in Fig. 1 (b).

Owing to the large plasmon energy, the experimental observation of plasmonic polaron bands in silicon may be hindered by the low resolution of ARPES measurements well below the Fermi energy. In order to identify materials in which such polaron bands may be observed, in the following we focus on group IV transition metal dichalcogenides $\left(\mathrm{MoS}_{2}, \mathrm{WS}_{2}, \mathrm{MoSe}_{2}\right.$, and $\left.\mathrm{WSe}_{2}\right)$. The EELS spectra of the three-dimensional parent compounds exhibit two distinct features around $\sim 8 \mathrm{eV}$ and $\sim 22 \mathrm{eV}$, corresponding to the excitation of $\pi$ and $\pi+\sigma$ plasmons, respectively [37, 38]. Since the width of the bands arising from transition metal $d$ states and chalcogen $p$ states in these compounds is approximately $7 \mathrm{eV}$ [39, possible plasmonic polarons are expected to appear between $\sim 8-15 \mathrm{eV}$. In this energy range the deep S-3s or Se- $4 s$ bands, located between 12-15 eV [39, dominate the spectral function, thereby hindering the identification of plasmonic polarons in ARPES also in this case.

At variance with this scenario, in the case of monolayer TMDs both experimental [40, and theoretical 41] studies reported plasmonic peaks in the EELS spectra which are strongly red-shifted with respect to their bulk counterpart. For example, in the case of $\mathrm{MoS}_{2}$ and $\mathrm{WS}_{2}$ monolayers the $\pi$ plasmons are found at energies around $6 \mathrm{eV}$. We thus expect to observe plasmonic polarons at binding energies between $6-13 \mathrm{eV}$. Since this energy win- dow matches the band structure gap between the metal$d /$ chalcogen- $p$ bands and the chalcogen $s$ bands, such plamonic polarons should be distinctly observable.

To examine this possibility on quantitative grounds, we calculate the $G W+\mathrm{C}_{\mathrm{AHK}}$ spectral functions of TMDs from first principles 42 . In order to contain the computational cost we describe the screening by introducing a two-pole approximation for the inverse dielectric matrix, as discussed in the Supplemental Material [34.

Figure 2(a) shows the complete $G W+\mathrm{C}_{\mathrm{AHK}}$ spectral function of monolayer $\mathrm{MoS}_{2}$ evaluated along the $\Gamma-M$ high-symmetry line. At binding energies between $0-5 \mathrm{eV}$ and 10-15 eV, the spectral function of monolayer $\mathrm{MoS}_{2}$ exhibits the standard quasi-particle peaks. As in the case of silicon, in monolayer $\mathrm{MoS}_{2}$ plasmonic polarons introduce new spectral features in a binding energy range where quasi-particle states are absent. To characterize these new features, we analyze their energy vs. wavevector dispersions using a Lorentzian decomposition of the energy profiles. This analysis allows us to disentangle the contributions of plasmonic polarons from the quasiparticle excitations. Figures 2(b) and (d) show the plasmonic polarons corresponding to the emission of a photoelectron and the simultaneous excitation of a $\pi$ or $\pi+\sigma$ plasmon, respectively. As for silicon, plasmonic polarons exhibit a clear energy-momentum dispersion relation that leads to the formation of blue-shifted replica of the valence band structure. In particular we find two replicas of the valence bands of monolayer $\mathrm{MoS}_{2}$, one associated with the $\pi$ plasmon centered around the binding energy $\sim 8 \mathrm{eV}$, and another one associated with the $\pi+\sigma$ plasmon around $\sim 19 \mathrm{eV}$. 
Owing to the approximate treatment of electron recoil effects in the cumulant approach [4, 18, the actual broadening might be even larger than in the present calculations. In order to understand whether plasmonic polaron bands could be observed in ARPES experiments it is therefore essential to quantify their spectral weight. A reliable measure of the intensity of the plasmonic bands can be obtained from the average number $a_{n \mathbf{k}}$ of plasmons emitted during the photoemission process 32] $a_{n \mathbf{k}}=\int \omega^{-2} \beta_{n \mathbf{k}}(\omega) d \omega$. In the case of the high-lying Mo- $4 d / \mathrm{S}-3 p$ bands we obtain $a_{n \mathbf{k}}$ in the range $0.08-0.12$ for momenta along the $\Gamma-M$ line, whereas $a_{n \mathbf{k}}$ is found in the range 0.04-0.06 for the lower-lying $\mathrm{S}-3 s$ bands. These results indicate that the formation of plasmonic polarons provides an important dissipation channel for the ARPES photo-current. In particular, in the case of Mo- $4 d_{z^{2}}$ states at the top of the valence band (the most important for electron transport in p-doped $\mathrm{MoS}_{2}$ ) every photo-hole is accompanied by 0.13 plasmons per unit cell. These estimates are confirmed by a direct integration of the spectral function, which shows that plasmonic polarons carry on average $12 \%$ of the total spectral weight of the valence electronic states. For the valence states the intensity ratio between the plasmonic polaron band and the quasi-particle peak is $\sim 0.01-0.02$. In addition, by considering the metal- $d /$ chalcogen- $p$ bands altogether we find that, in the case of monolayer $\mathrm{MoS}_{2}$, it should be possible to extract up to $1.2 \cdot 10^{14}$ electrons $/ \mathrm{cm}^{2}(0.1$ electrons/cell) at binding energies inside the band structure gap between Mo-4d/S-3p bands and S-3s bands.

At variance with the case of monolayer $\mathrm{MoS}_{2}$, for the bulk compound our calculations indicate that the plasmonic bands overlap substantially with the deep S$s$ states (Fig. 3). The overlap between ordinary quasiparticle bands and plasmonic polaron bands should make the experimental detection of these new features in bulk $\mathrm{MoS}_{2}$ much more challenging than in the case of a monolayer.

The above results suggest that two key conditions need to be satisfied for plasmonic polaron band structures to be clearly observed in ARPES: (i) the existence of lowenergy plasmon excitations in the EELS spectrum, and (ii) the presence of a band gap in the valence band manifold. The condition (ii) also contributes to minimize spectral broadening arising from electron-phonon scattering.

Given these 'design rules' it is natural to ask whether there exist 'optimal' TMDs for observing plasmonic polaron bands. To answer this question, we repeated our $G W+\mathrm{C}_{\mathrm{AHK}}$ calculations for the related monolayer compounds $\mathrm{MoSe}_{2}, \mathrm{WS}_{2}$, and $\mathrm{WSe}_{2}$. Figure 3 shows that plasmonic polaron bands associated with $\pi$ plasmons fit inside the band gap betwen the metal-d/chalcogen$p$ bands and the chalcogen- $s$ bands for all these compounds. In particular we find that monolayer $\mathrm{MoSe}_{2}$ should provide an ideal testbench for identifying plas-

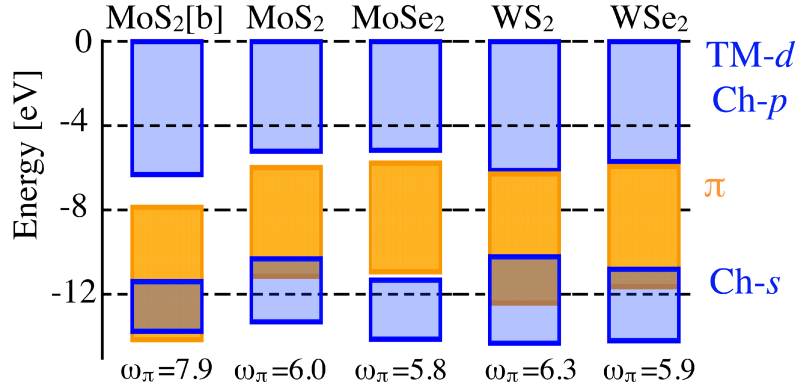

Figure 3. Energy range of the quasi-particle bands (blue boxes) and the $\pi$-plasmonic polaron bands in monolayer $\mathrm{MoS}_{2}, \mathrm{MoSe}_{2}, \mathrm{WS}_{2}$, and $\mathrm{WSe}_{2}$, evaluated within the $G W+\mathrm{C}_{\mathrm{AHK}}$ approach (yellow boxes). Bulk $\mathrm{MoS}_{2}$ is included for comparison $\left(\mathrm{MoS}_{2}[\mathrm{~b}]\right)$. The topmost bands arise from the hybridization of transition metal $d$ states and the chalcogenide $p$ states (TM- $d / \mathrm{Ch}-p$ ); the low-energy ones from the chalcogenide $s$ states $(\mathrm{Ch}-s)$. The plasma energies $\omega_{\pi}$ are given in $\mathrm{eV}$.

monic polarons, since in this case the plasmonic structures exhibit essentially no overlap with the ordinary valence bands (Fig. 3).

Since the plasmon energy scales with square root of the static dielectric constant $\epsilon_{0}$, it should also be possible to realize plasmonic band structure engineering in TMD monolayers by modifying their dielectric screening properties. For example this could be achieved by using different substrates, by building van der Waals heterostructures of TMDs 43, using doping 44, or mechanical deformation [45, 46]. All these modifications would leave the ordinary valence band structure essentially unaffected (on the eV scale), while producing significant shifts in the binding energy of plasmonic polarons.

In conclusion, using state-of-the-art first-principles $G W$ plus cumulant calculations, we have shown that electron-plasmon coupling leads to the formation of plasmonic polaron band structures, and that group IV transition metal dichalcogenide monolayers (in particular monolayer $\mathrm{MoSe}_{2}$ ) should provide a unique opportunity for observing these new features in ARPES experiments. Remarkably, we have found that these plasmonic band structures exhibit dispersion relations which closely follow the ordinary valence bands and, similarly to quasiparticle bands, lead to the formation of Van Hove singularities in the density of states. More generally, plasmonic-polaron bands emerge as novel spectroscopic signatures of electron-plasmon coupling, which may contribute to unravel the complexity of ARPES measurements. The emergence of plasmonic polarons is not limited to semiconductors, and may also prove useful to rationalize the electronic structure of materials characterized by well defined plasmonic excitations, including for example metals, as well as $d$ - and $f$-electron systems [47, 48. In fact, our work raises the question on whether 
the concept of plasmonic polarons may be systematically employed in the interpretation of ARPES spectra of complex systems. As an example, while our work focused on the simplest case of 'isolated' plasmonic polaron bands, the crossing of plasmonic bands and high-lying valence bands may reveal the emergence of band-branching effects, in analogy with the polaron problem in electronphonon physics [49].

This work was supported by the Leverhulme Trust (Grant RL-2012-001) and the European Research Council (EU FP7 / ERC grant no. 239578 and EU FP7/grant no. 604391 Graphene Flagship). Calculations were performed at the Oxford Supercomputing Centre and at the Oxford Materials Modelling Laboratory.

[1] A. Damascelli, Z. Hussain, and Z.-X. Shen, Rev. Mod. Phys. 75, 473 (2003).

[2] Y. Liang and L. Yang, Phys. Rev. Lett. 114, 063001 (2015)

[3] S. A. Maier and H. A. Atwater, J. Appl. Phys. 98, 011101 (2005).

[4] L. Hedin, Phys. Scr. 21, 477 (1980)

[5] P. Nozières and D. Pines, Phys. Rev. 113, 1254 (1959)

[6] A. Lanzara et al., Nature 412, 510 (2001)

[7] A. Bostwick et al., Nat. Phys. 3, 36 (2007).

[8] F. Giustino, M. L. Cohen, and S. G. Louie, Nature 452, 975 (2008)

[9] T. Dahm et al., Nat. Phys. 5, 217 (2009).

[10] S. Moser et al., Phys. Rev. Lett. 110, 196403 (2013)

[11] J. P. Carbotte, T. Timusk, and J. Hwang, Rep. Prog. Phys. 74, 066501 (2011).

[12] B. Lundqvist, Phys. Kondens. Mater. 6, 193 (1967)

[13] D. C. Langreth, Phys. Rev. B 1, 471 (1970).

[14] S. Engelsberg and J. R. Schrieffer, Phys. Rev. 131, 993 (1963)

[15] We purposely avoid the term "plasmarons" introduced by Lundqvist [12]. In the original formulation the plasmaron was understood as a new elementary excitation resulting from a pole of the electron's Green's function. Subsequent analysis by Langreth 13 showed that the poles identified in 12 are an artifact of the $G W$ approximation, but there exists a polaron resonance in analogy with the standard electron-phonon theory [4, 13].

[16] G. Mahan, Many-Particle Physics (Springer, 2000).

[17] F. Aryasetiawan, L. Hedin, and K. Karlsson, Phys. Rev. Lett. 77, 2268 (1996)

[18] M. Guzzo et al., Phys. Rev. Lett. 107, 166401 (2011)

[19] A. S. Kheifets et al., Phys. Rev. B 68, 233205 (2003)

[20] A. Bostwick et al., Science 328, 999 (2010)

[21] M. Polini et al., Phys. Rev. B 77, 081411 (2008)

[22] L. Hedin, Phys. Rev. 139, A796 (1965).

[23] M. S. Hybertsen and S. G. Louie, Phys. Rev. B 34, 5390 (1986)

[24] G. Onida, L. Reining, and A. Rubio, Rev. Mod. Phys. 74, 601 (2002)

[25] B. Holm and F. Aryasetiawan, Phys. Rev. B 56, 12825 (1997)
[26] J. Lischner, D. Vigil-Fowler, and S. G. Louie, Phys. Rev. Lett. 110, 146801 (2013)

[27] M. Guzzo et al., Eur. Phys. J. B 85, 324 (2012)

[28] J. J. Kas, J. J. Rehr, and L. Reining, Phys. Rev. B 90, $085112(2014)$

[29] M. Guzzo et al., Phys. Rev. B 89, 085425 (2014).

[30] B. Radisavljevic et al., Nat. Nanotechnol. 6, 147 (2011)

[31] Q. H. Wang et al., Nat. Nanotechnol. 7, 699 (2012).

[32] L. Hedin, J. Phys.: Condens. Matter 11, R489 (1999).

[33] F. Bruneval, N. Vast, and L. Reining, Phys. Rev. B 74, 045102 (2006)

[34] See Supplemental Material at tobeaddedbypublisher which includes Refs. 50 53 .

[35] F. Giustino, M. L. Cohen, and S. G. Louie, Phys. Rev. B 81, 115105 (2010)

[36] H. Lambert and F. Giustino, Phys. Rev. B 88, 075117 (2013)

[37] W. Y. Liang and S. L. Cundy, Philos. Mag. 19, 1031 (1969)

[38] M. Bell and W. Liang, Adv. Phys. 25, 53 (1976)

[39] P. Raybaud et al., J. Phys.: Condens. Matter 9, 11107 (1997)

[40] J. N. Coleman et al., Science 331, 568 (2011).

[41] P. Johari and V. B. Shenoy, ACS Nano 5, 5903 (2011)

[42] Ground-state density-functional theory calculations in the local density approximation (LDA) [54, 55] are performed using the Quantum-ESPRESSO software package [56]. We used normconserving Hartwigsen-GoedekerHutter pseudopotentials and a 29 Ry kinetic energy cutoff, which suffices for converging the quasiparticle energies of $\mathrm{MoS}_{2}$ [57. The Brillouin zone is sampled using a $20 \times 20$ Monkhorst-Pack grid. For monolayer $\mathrm{MoS}_{2}$ we employed a supercell approach with a $16.4 \AA$ A interlayer spacing between periodic replicas. In the $G W+\mathrm{C}_{\mathrm{AHK}}$ spectral function [Eqs. [13] ] we neglect the asymmetry factor [4] and the exchange part of the quasi-particle correction as we are primarily interested in the intensity and binding energy of the spectral features. Sternheimer- $G W$ calculations for silicon employ the same computational parameters as in 36.

[43] A. K. Geim and I. V. Grigorieva, Nature 499, 419 (2013)

[44] B. Radisavljevic and A. Kis, Nat. Mater. 12, 815 (2013)

[45] A. Castellanos-Gomez et al., Nano Lett. 13, 5361 (2013)

[46] H. Peelaers and C. G. Van de Walle, Phys. Rev. B 86, $241401(2012)$

[47] M. Gatti and M. Guzzo, Phys. Rev. B 87, 155147 (2013)

[48] S. Basak et al., Phys. Rev. B 80, 214520 (2009)

[49] A. Eiguren, C. Ambrosch-Draxl, and P. M. Echenique, Phys. Rev. B 79, 245103 (2009)

[50] R. W. Godby and R. J. Needs, Phys. Rev. Lett. 62, 1169 (1989)

[51] G. E. Engel and B. Farid, Phys. Rev. B 47, 15931 (1993)

[52] S. Baroni et al., Rev. Mod. Phys. 73, 515 (2001).

[53] A. Frommer, Computing 70, 87 (2003).

[54] W. Kohn and L. J. Sham, Phys. Rev. 140, A1133 (1965).

[55] P. Hohenberg and W. Kohn, Phys. Rev. 136, B864 (1964)

[56] P. Giannozzi et al., J. Phys.: Condens. Matter 21, 395502 (2009).

[57] F. Hüser, T. Olsen, and K. S. Thygesen, Phys. Rev. B 88, 245309 (2013). 